\title{
Energy Conscious Routing in MANET
}

\author{
Namita Agrawal \\ Student, \\ Computer Department, \\ MPSTME, \\ NMIMS, Shirpur, Dist :Dhule, \\ Maharashtra, India
}

\author{
Nitin Choubey \\ Faculty, \\ Computer Department, \\ MPSTME, \\ NMIMS, Shirpur, Dist :Dhule, \\ Maharashtra, India
}

\author{
Prashant Mishra \\ Faculty, \\ Computer Department, \\ MPSTME, \\ NMIMS, Shirpur, Dist :Dhule, \\ Maharashtra, India
}

\begin{abstract}
The Mobile ad hoc network (MANET) are infrastructure-less, self-organizing, rapidly deployable wireless networks, they are highly suitable for applications involving special outdoor events, communications in regions with no wireless infrastructure, emergencies and natural disasters, and military operations. Routing is one of the key issues in MANETs due to their highly dynamic and distributed nature. In particular, energy efficient routing is the most important design criteria for MANETs since mobile nodes will be powered by batteries with limited capacity. The routing mechanism is based on minimum hop counts, the distance between each pair of nodes becomes larger and hence higher transmission power is required for the communication. Power failure of a mobile node not only affect the node itself but also its ability to forward packets on behalf of others and thus the overall network lifetime. The low residual energy might be one of the reasons of route breakage. Also the mobility of nodes makes link failure and retransmission of packet. In this paper we have modified the Energy Conscious DSR protocol which has basic protocol Dynamic Source routing (DSR). The paper focuses on the mobility of nodes, low residual energy which is the cause of route breakage and packet retransmission, which get solved and communication is keep going without any interruption and the network lifetime increases. We have validated our proposed protocol through ns-2.34 and evaluated the performance of the networks by considering few energy metrics. We got the results as our modified ECDSR outperforms DSR and Ad-Hoc on demand Distance Vector (AODV) in performance analysis.
\end{abstract}

\section{General Terms}

Mobile Ad-Hoc Network, Routing algorithm, Dynamic Source Routing

\section{Keywords}

MANET, Energy efficiency, AODV, DSR, ECDSR.

\section{INTRODUCTION}

Ad-hoc wireless network is a collection of two or more devices equipped with wireless communication and networking capability. Mobile Ad Hoc Network (MANET) is communication network in which all nodes are mobile and communicate with each other via wireless connections. Nodes can join or leave the network at any time and they communicate with each other which are immediately within their radio range and communication beyond this range is established by employing intermediate nodes to set up a path in a hop-by-hop manner. There is no fixed infrastructure. There is no need for any fixed radio base stations, any wires or fixed routers. All nodes are equal and there is no centralized control or overview that is it is self-organizing and adaptive. This means that a formed network can be de-formed on-the-fly without need for any system administration. There are no designated routers: all nodes can serve as routers for each other, and data packets are forwarded from node to node in a multi-hop fashion. Due to presence of mobility, routing information will have to change to reflect changes in link connectivity. The diversity of ad hoc mobile devices also implies that the battery capacity of such devices will also vary. Since ad hoc networks rely on forwarding data packets sent by other nodes, power consumption becomes critical issue. [1][3][9].

\subsection{Routing Protocols Energy Efficiency In MANET}

Routing is the process of selecting paths in a network along which to send the important data. A major challenge that a routing protocol designed for ad hoc wireless networks faces is resource constraints. Energy efficiency is a major issue of concern in wireless an ad hoc networks as mobile nodes rely on batteries, which are limited sources of energy, and, in many environments, it is quite a cumbersome task to replace or recharge them. Despite the progress made in battery technology, the lifetime of battery powered devices continues to be key challenge and requires additional research on efficient design of platforms, protocols, and systems. Nodes within an ad hoc network generally rely on batteries (or exhaustive energy sources) for power. Since these energy sources have a limited lifetime, power availability is one of the most important constraints for the operation of the ad hoc network. There are different sources of power consumption in a mobile node. Communication is one of the main sources of energy consumption. Since the rate of battery performance improvement is rather slow currently, and in the absence of breakthroughs in this field, other measured have to be taken to achieve the goal of getting more performance out of the currently available battery resources.

Devices used in the mobile ad hoc networks require portability and as they are mobile they also have size and weight constraints along with the restrictions on the power source. If the battery power is increased, it may make the nodes bulky and less portable. So the energy efficiency remains an important design consideration for these types of networks. A major challenge that a routing protocol designed for mobile ad hoc wireless network faces is resource constraints. Therefore mobile ad hoc routing protocol must optimally balance these conflicting aspects.

To achieve the desired behaviour, so many energy efficiency mechanisms have been proposed. None of them can perform better in every condition. It depends upon the network parameters which decide the protocol to be used. Some 
proposals make use of clustering or maintain multiple paths to destinations (in order to share the routing load among different nodes). The majority of energy efficient routing protocols for MANET try to reduce energy consumption by means of an energy efficient routing metric, used in routing table computation instead of the minimum-hop metric. This way, a routing protocol can easily introduce energy efficiency in its packet forwarding. These protocols try either to route data through the path with maximum energy bottleneck, or to minimize the end-to-end transmission energy for packets, or a weighted combination of both. The energy optimization of a routing protocol, however, can exploit also other network layer mechanisms, like control information forwarding. [4][5]

So for our paper, we have considered the basic mechanism of one of the well known routing protocol that is Dynamic Source Routing (DSR) protocol. We have chosen DSR because it has been accepted as a better routing protocol with compare to any other protocols like AODV, TORA by considering various performance metrics. We have made an attempt to modify this DSR as an Energy conscious DSR (ECDSR).

So the next section 2 will explain mechanism for AODV. The section 3 shades some lights on basic DSR mechanism. Then in section 4 we have explained the proposed method that is modified ECDSR and its mechanism followed by algorithm in section 5. Then in the section 6 we have analyzed results of the performed simulation and concluded the result of algorithm .in the section 7 .

\section{AD-HOC ON-DEMAND DISTANCE VECTOR (AODV)}

AODV is the enhancement of DSDV (Destination Sequenced Distance Vector). But the basic difference between both is that AODV is the reactive routing protocol whereas DSDV is the proactive routing protocol. AODV is based on the hop to hop routing methodology. It is basically the combination of on-demand and distance vector routing protocol.

\subsection{Procedure}

Firstly, when the node wants to know the route from source to destination it first of all creates the ROUTE REQUEST mechanism. Next, when the route request is forwarded by intermediate nodes it also creates a reverse route for itself for Destination. When the request reaches a node with route to destination it creates a ROUTE REPLY mechanism which contains the number of hops that are required to reach the destination. All nodes that take part in forwarding this route reply packet to the source node creates a forward route to destination. When the node forward packets, it type in ID in its table so that it constructs route from destination node to source node. If the source node moves, it will start route discovery again. If the intermediate node moves, the link will be invalid and the node will send this message to source nodes. Then, the source node will start route discovery again. The route created by each node from source to destination is by hop-to-hop methodology. [11]

\section{DYNAMIC SOURCE ROUTING (DSR)}

In MANET basically the routing protocols has divided into two categories, i. Proactive and ii. Reactive protocols. In the Proactive protocols every node maintains the route for the entire network and in the Reactive protocols a route to a certain destination has been created only when the source node is demanding for a particular destination. DSR comes under reactive protocol and among all the reactive protocols
DSR is a well-known protocol. It firstly performs two basic operations during its whole routing process, which are route discovery and route maintenance. If a node wants to send a packet to a destination, then prior to that it has to check its own routing cache that there exists any route available to that destination or not. If it gets a route, then it sends the packet to the destination. Otherwise it starts the route discovery phase.

\subsection{Route Discovery}

In this phase, when a source has some data packet to send then it starts looking for a route from source node to destination node. Also when the node wishes to contact destination node which is not in transmission range so it should obtain a route by launching the Route Discovery Mechanism. As every node is having its own route cache, the source node first checks its own route cache and if it has no route to the destination then it goes for another two sub processes, i. Route Request and ii. Route Reply.

\subsubsection{Route Request}

If in the route cache of the source node, the route is not found, then the source node broadcasts a Route Request (RREQ) message to all its neighbor nodes using flooding. The Route Request (RREQ) message contains the address of source node, destination address and a unique identification number. Due to this unique identification number each node processes that RREQ only once. To the route record of the packet each intermediate node appends its own address and keeps broadcasting the RREQ message until it finds the destination node in its own route cache during the RREQ propagation.

\subsubsection{Route Reply}

When the destination node receives the RREQ with same destination address in the RREP packet or being generated by any an intermediate node if the routing information about the destination is available in its own route cache in the above both conditions the route reply (RREP) message is generated. And in between that the sequence of hops are also kept updating in the RREQ packet. On the basis of minimum hop count, the best path is selected by the destination node. Also the destination node generates the RREP packet back to the source by keeping the route record from route request packet into the route reply packet.

In case of an intermediate node, when they generate the RREP message then the node appends its cached route to the destination to the route record in the RREQ packet and then generates the RREP packet. Finally, the source collects the route carried by the RREP that it receives for future use.

\subsection{Route Maintenance}

Due to dynamic nature of MANET, it should have specific mechanism to maintain the link from source to destination. A network partition may take place if a single node participating in the routing process comes out of the range to its neighbor node at any instance. A network partition increases the packet loss as well as it affects the increase in few costly operations like route discovery, route maintenance, path repair and many more. By generating route error message or some acknowledgement the route maintenance process is performed. So during sending the packets if some trouble occurs in link the process route maintenance acts as a route repair.

\section{ENERGY CONSCIOUS DSR (ECDSR)}

The main goal of ECDSR is to select the path between the specified source and destination in such a way that at a given time all intermediate nodes will have higher level of energy. 
So the route discovery phase from DSR gets changed in ECDSR. So during the route discovery phase, select that path whose intermediate nodes are having higher remaining battery power instead of following minimum hop count method which is in DSR. During communication if the energy level of any node reaches to the minimum threshold value, a new path will be chosen in order to continue the communication without any interruption. So for the low energy node the modified algorithm has not only introduced an energy saving feature but also introduced an energy survival characteristic. Hence the proposed algorithm is more likely to be energy conscious while establishing a connection through many intermediate nodes. So ECDSR has two main phases, i. ECDSR Energy Saving Phase and ii. ECDSR Energy Survival Phase.

\subsection{ECDSR Energy Saving Phase}

This mechanism is applied during the route discovery phase when the source node has some packets to send to the destination. This phase has the most energy efficient path among all possible paths, so that the communication can be carried out for a long period of time. So the route discovery phase of routing has been changed to maximum energy conscious path carrying higher energy intermediate nodes in ECDSR from minimum hop count of traditional DSR.

In the traditional DSR during route discovery process if any node receives a Route Request (RREQ) which is not to be meant for it or if the receiving node is not the final destination then it holds the packet for a certain time interval which has been selected pseudo-randomly from a uniform distribution of probability between 0 to 0.01 . Usually 0.01 is considered as constant broadcast jitter, after keeping the packet for that amount of time, it then broadcasts the packet.

But in ECDSR, there is a use of a kind of dynamic jitter for the control packets like RREQ and RREP. And the method of using different value of jitter depends on the remaining residual battery power of the intermediate nodes. The value of jitter has not been disturbed as the range of jitter varies from 0 to $0.01 \mathrm{sec}$. The more will be residual power of the intermediate nodes, the lesser amount of delay will be imposed on the control packets. If any node is having low energy than certain threshold value, then it will avoid itself from participating as an intermediate node and sends an error message to the source. The delay in this proposed method is inversely proportional to the residual battery power. As in the traditional DSR, the pseudo-random delay is maximum that is $0.01 \mathrm{sec}$. But, ECDSR has used a special formula to calculate the delay. If the energy of a node is more than the minimum level of energy that is 1 Joule (low energy), then the delay should be less than $0.01 \mathrm{sec}$. Otherwise the delay will be maximum that is $0.01 \mathrm{sec}$. The minimum value of the energy is set to 1 joule and it corresponds to the delay of $1 /(1 * 100)$ $=0.01 \mathrm{sec}$. In our simulation we have set the initial energy of the node as 100 Joules. So the delay introduced at RREQ packet is $1 /(100 * 100)=0.0001 \mathrm{sec}$. So the new path in ECDSR contains the path with maximum some of total remaining energy of the intermediate nodes which in turn provides a higher possibility of a longer communication without any interruption of link breakage unless the any intermediate node moves out of the range of its neighbor nodes. So with the above approach, the RREQ packet will reach at the destination which has maximum sum of remaining energy, because delay is inversely proportional to the remaining residual battery power.

\subsection{ECDSR Energy Survival Phase}

During the route maintenance process, the energy survival phase comes out in action. In DSR, due to low residual energy power the link lost or retransmission of packet is never been considered. Either congestion or node mobility was taken as one of the reason for packet lost or route lost at any time during communication. As in previous experiment authors assumed their network to be static one, so they have neglect the node mobility possibility which may be one of the reasons of path lost or packet retransmission. In our extended experiment the network used is dynamic one, so the mobility is also used. But in ECDSR energy survival method, an additional cause is included where low residual energy might be one of the reasons of route breakage. And through this method energy consumption is get balanced in all intermediate nodes.

As all intermediate nodes get used continuously unless communication gets over, the residual battery power is getting depleted continuously. So the moment any intermediate node finds its own residual energy level below or equal to a specific threshold value, then certainly it will inform to all its neighbor nodes that it is no more ready either to receive or to forward the packet. This can be informed through an alert message generated by the same node and insists to the source node to find another node for the same destination. So we have set the minimum threshold value as 1 Joule, so once any intermediate node has its remaining energy as 1 joule, it means it will set a "Low Energy" field to be 1 and this field is to be added with the error control message. The moment neighbor nodes receive this error message with Low Energy field with 1, and then they will simply discard the route containing the affected node. And once this error message reaches at source, and then the source starts looking for a new path present in its route cache, otherwise it will go for a route discovery process. So in this way, a node informs the source node to find a new path before any route breakage occurs and hence the established communication continues without any interruption. One other major advantage of this energy survival is to save the node from getting dead completely. It keeps the low energy node alive which can also be used in some other crucial communication in future. In this way for our experiment the mobility of nodes is considered as well as low residual energy which is the cause of route breakage and packet retransmission is solved and communication is keep going without any interruption.[6][7]

\section{ENERGY CONSCIOUS DSR (ECDSR)}

While developing this algorithm let assumed that the nodes that are participating in the communication do not need to have equal initial battery power. Because we cannot assure that the node participating for a communication is not being used earlier.

1) Processing of route request by source node:

To find the efficient route, the route request is broadcasted to the medium. The nodes which receive the route request packet compute the delay and add it to the packet header. So the route request can be processed either by the destination node or intermediate nodes.

2) Processing of the route request by destination node:

The node checks whether RREQ is first arrived by looking up the sequence number and source node id in cache. If RREQ is first arrived, the destination node sends a "Route Reply" to the initiator of the route request packet in which it includes the entire source route from the initiator to the destination. And then it will discard the other route request having the same source node or if the route request is having the same 
sequence number. The destination node will treat the route request as the first come first serve basis.

3) Processing of the route request by destination node:

It checks whether RREQ is new by looking up the sequence number and source node id in cache as like in target nodes. If the RREQ is new one then it calculates the delay and includes it in the packet header and after keeping the packet till the calculated delay time it forwards to its neighbor node. If the destination node is in the cache then simply it uncast the RREQ for checking. If the destination node is not in its route cache then it again broadcast the RREQ to its neighbor nodes. 4) Processing of error control packets:

Whenever the energy of any node reaches less than or equal to 1 Joule then "Low Energy" field in the DSR header packet will be set to 1 and it is broadcasted to it's all neighbour nodes. When the neighbor nodes of the affected node receive the error packet, then they will remove the path containing the affected node from its route cache and broadcast an error (Route Error) to the source. As soon as the error message received by the source node, it searches the path which is present in its route cache or else it triggers the route discovery phase. [6][7]

\section{EXPERIMENTAL RESULTS}

We have used the network simulator-2.34 [8] [12] to perform our experiment. We have included the Energy Model as well as the Two Ray Ground Model to calculate the residual battery power and to fix up the transmission power at every node. We set up a dynamic network consists of a test bed of $50,60,70,80,90$ nodes confined in a $1000 \mathrm{~m} \times 1000 \mathrm{~m}$ in square area. All nodes are assigned with the number from node- 0 to Node $\mathrm{N}-1$. We have set initial battery power for all nodes are having 100 joules of initial battery energy. The length of simulation time is $200 \mathrm{sec}$. We have introduced FTP traffic at node- 0 which is a source node. Below the list of parameter are shown in the simulation table 1 and the screenshots for different node densities are shown in following.

Table 1: Simulation Parameters

\begin{tabular}{|l|l|}
\hline Simulation Parameter & Value \\
\hline Network size & $1000 \mathrm{X} 1000$ \\
\hline Number of Nodes & $50,60,70,80,90$ \\
\hline Routing protocol & DSR \\
\hline Simulation Duration & $200 \mathrm{sec}$ \\
\hline Packet size & 2000 \\
\hline Queue Type & Drop Tail \\
\hline Propagation Model & Two Ray Ground \\
\hline Antenna & Omni antenna \\
\hline Transmission Range & 250 meters \\
\hline RxPower & 0.01 \\
\hline TxPower & 0.02 \\
\hline Initial Energy of Nodes & 100 Joules \\
\hline
\end{tabular}

\subsection{Experimental Results}

The simulation scenario for the different node densities that is $50,60,70,80,90$ nodes with mobility criteria is shown in figure $2,3,4,5,6$.

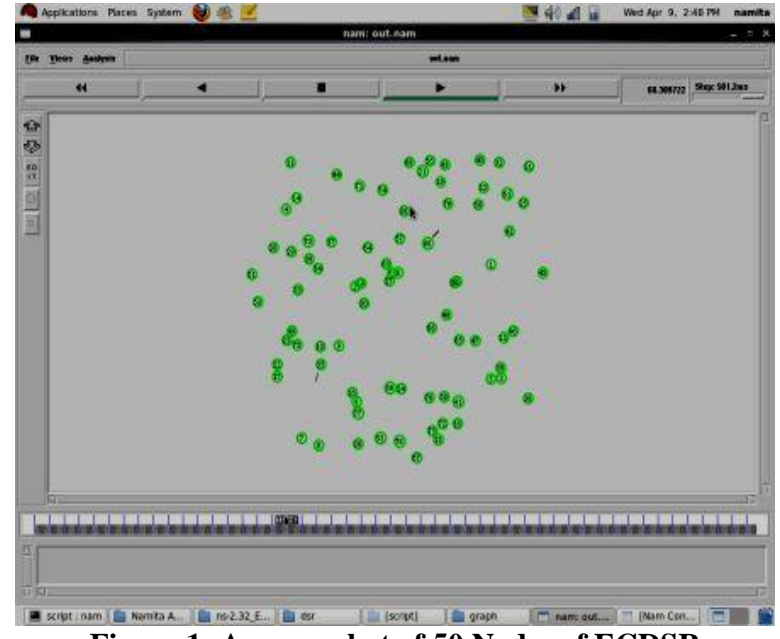

Figure 1: A screenshot of 50 Nodes of ECDSR

Above fig 1 shows the simulation for the 50 nodes for the ECDSR algorithm which produce the trace file containing all the routes for source to destination with energy of every node.

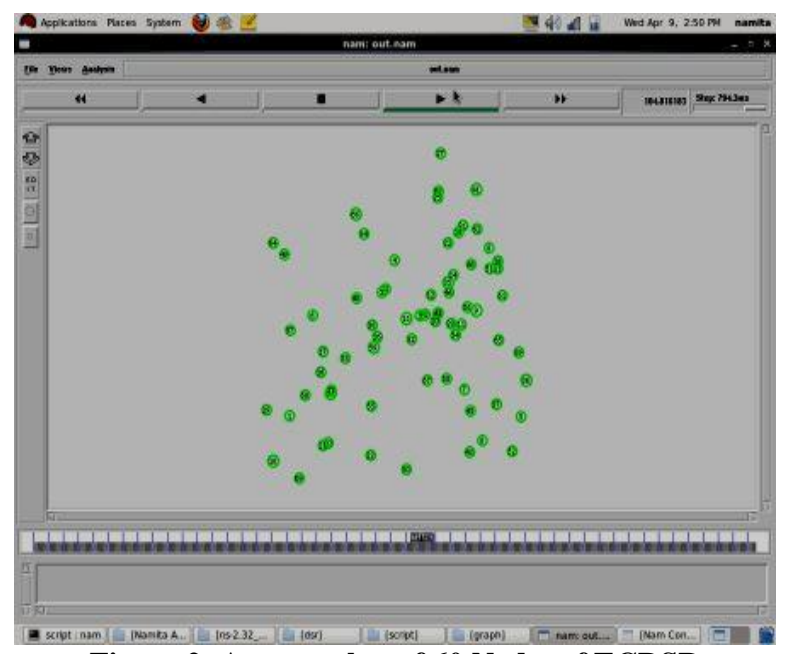

Figure 2: A screenshot of 60 Nodes of ECDSR

Above fig 1 shows the simulation for the 50 nodes for the ECDSR algorithm which produce the trace file containing all the routes for source to destination with energy of every node.

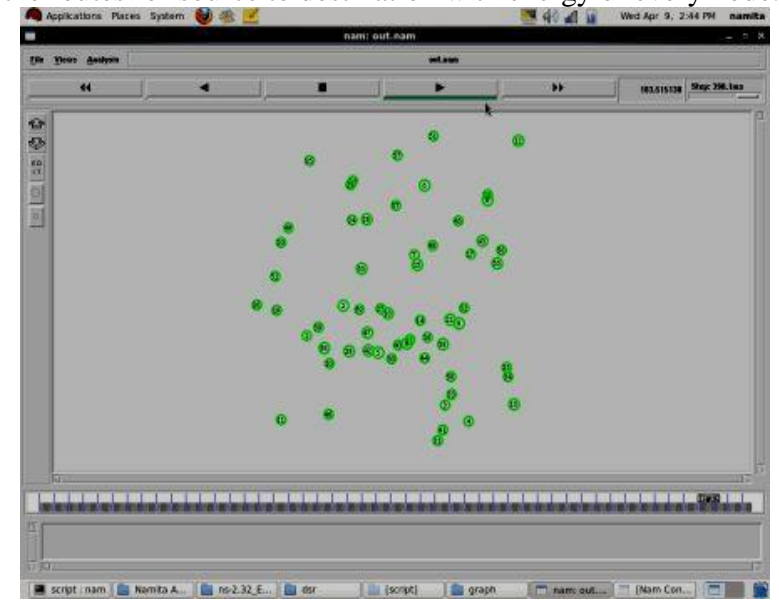

Figure 3: A screenshot of 70 Nodes of ECDSR

Above fig 1 shows the simulation for the 50 nodes for the ECDSR algorithm which produce the trace file containing all the routes for source to destination with energy of every node. 


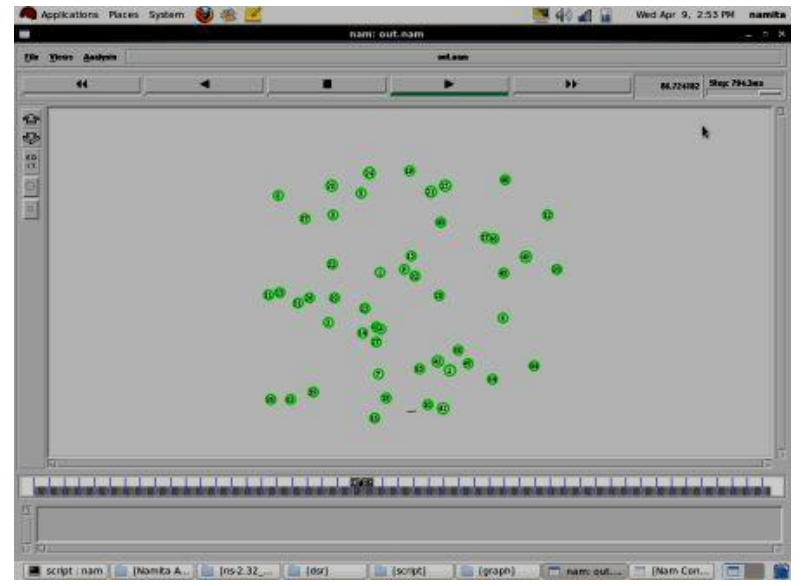

Figure 4: A screenshot of 80 Nodes of ECDSR

Above fig 1 shows the simulation for the 50 nodes for the ECDSR algorithm which produce the trace file containing all the routes for source to destination with energy of every node.

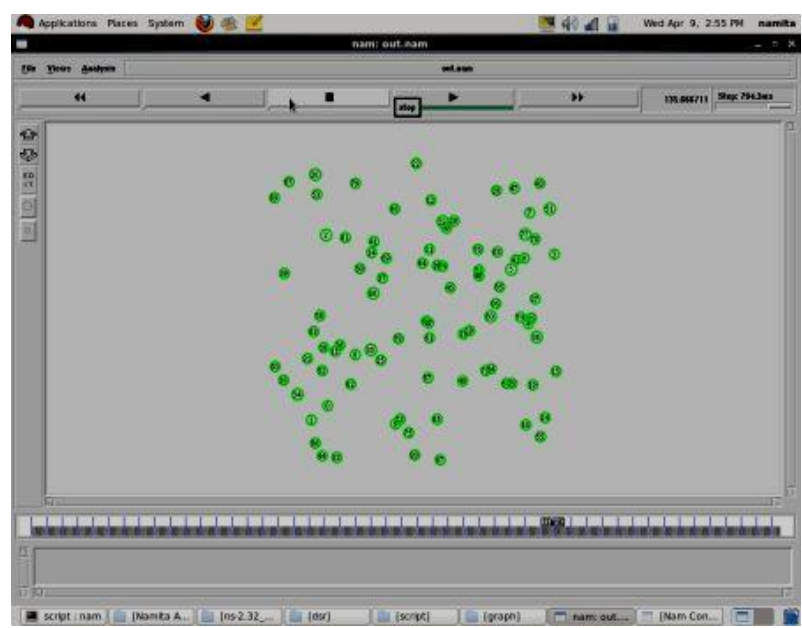

Figure 5: A screenshot of 90 Nodes of ECDSR

Above fig 1 shows the simulation for the 50 nodes for the ECDSR algorithm which produce the trace file containing all the routes for source to destination with energy of every node.

\subsection{Simulation Results}

\section{Packet delivery ratio:}

The packet delivery ratio is the ratio of number of delivered data packet to destination. The PDR gives the level of delivered data to destination. The greater the value of packet delivery ratio means the better performance of protocol.

Packet Delivery Ratio $=\sum$ Number of packets receive $/ \sum$ Number of packet send

The Packet Delivery ratio is that is packet deliver to the destination is higher in ECDSR as compared to DSR and AODV.

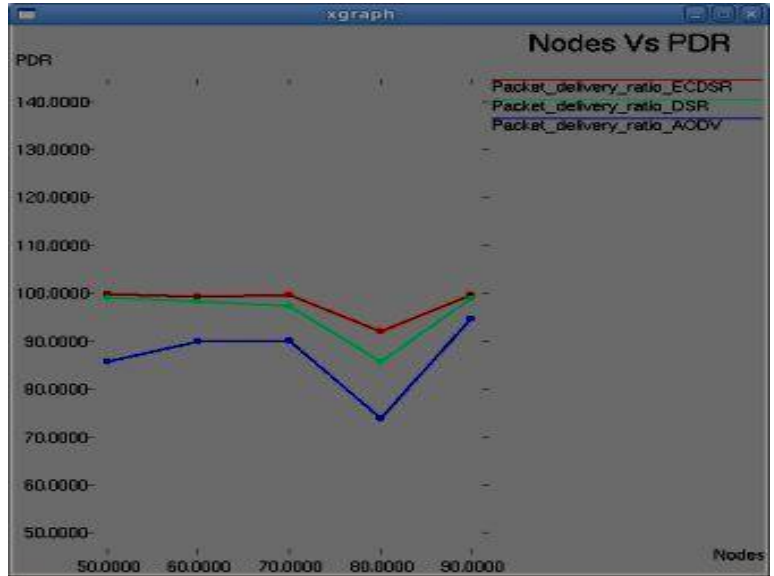

Figure 6: Number of nodes Vs Packet Delivery ratio

\section{Control Overhead:}

It is ratio of the control information sent to the actual data received at each node. The control information required for ECDSR is minimum as required to DSR and AODV.

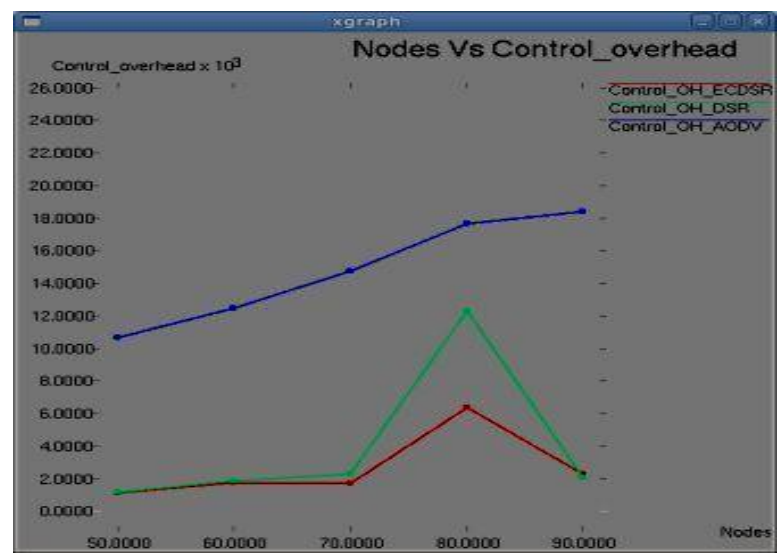

Figure 7: Number of nodes Vs Control overhead

\section{Normalized Routing Head:}

Normalized Routing Overhead is defined as the total number of routing packet transmitted per data packet.

Normalized Routing Overhead $=\sum$ number of routing packets sent (includes forwarded routing packets as well) $/ \sum$ number of data packets received.

The normalized routing head is less as compare with DSR in ECDSR and AODV.

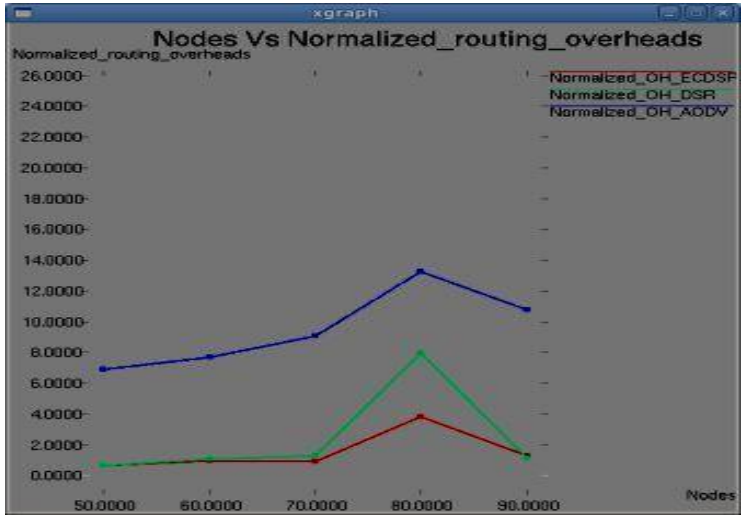

Figure 8: Number of nodes Vs Normalized Routing Overhead 


\section{Jitter:}

Jitter is the deviation of signals over a time period. Jitter and delay are related to each other. So from our experiment the jitter for ECDSR is low than DSR and AODV which is useful in routing.

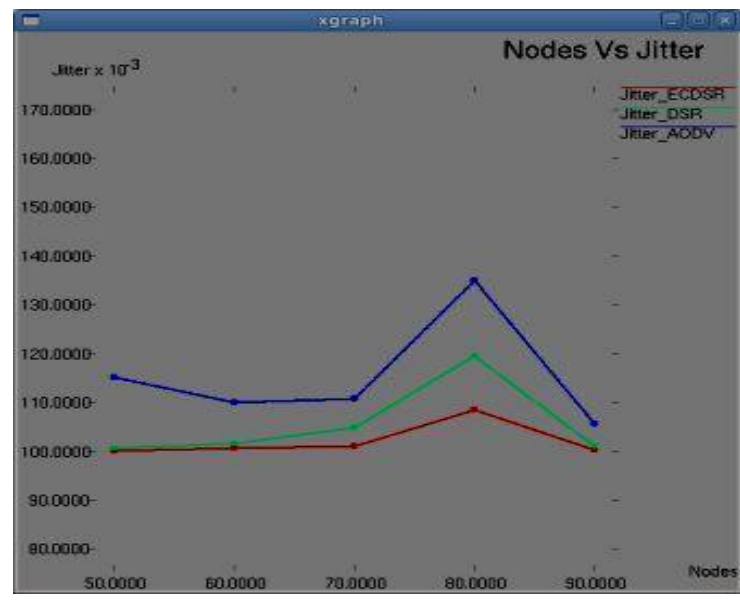

Figure 9: Number of nodes Vs Jitter

\section{Throughput:}

Throughput is the measure of how fast a node can actually sent the data through a network. So throughput is the average rate of successful message delivery over a communication channel. Throughput for the ECDSR is high than DSR and AODV. Also as Control overhead decreases the throughput get increased in ECDSR.

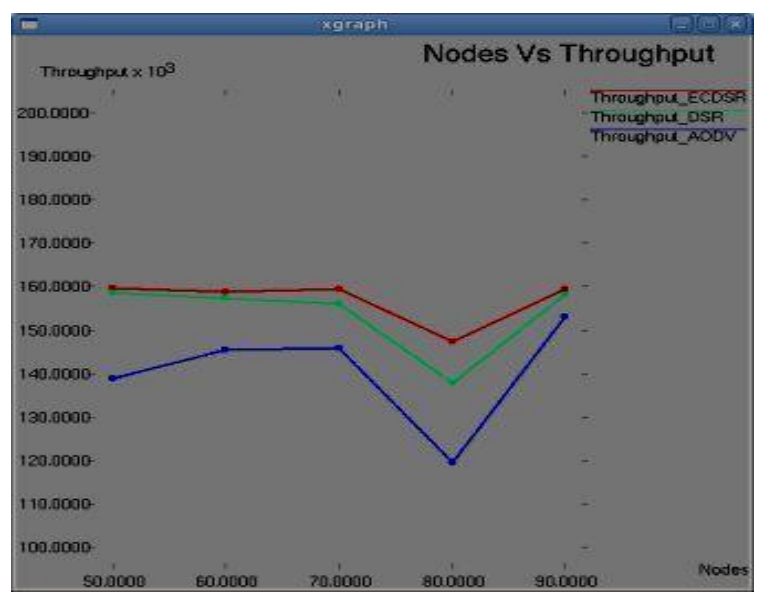

Figure 10: Number of nodes Vs Throughput

\section{Dropping Ratio:}

When a network has less number of dropping nodes then the reliability and the life time of network becomes higher. As in our experiment survival feature exists the low energy nodes do not take part in communication and high energy nodes are used to search path. The dropping ratio is less in ECDSR.

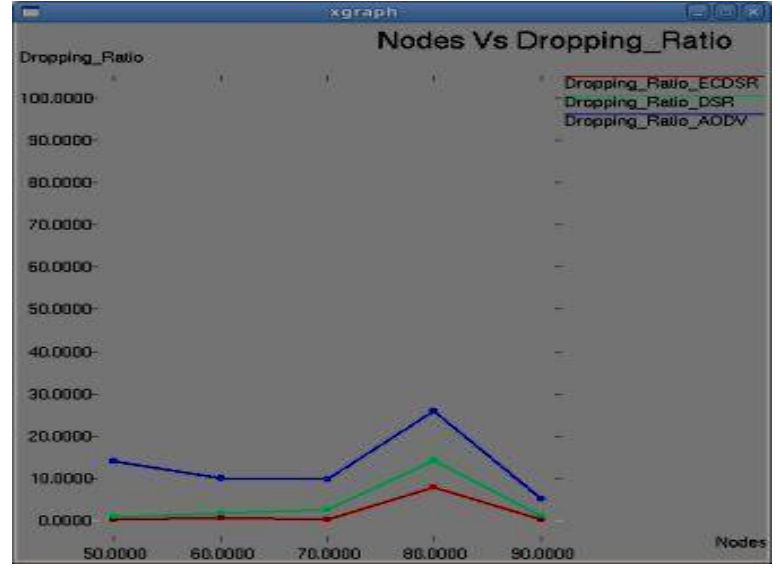

Figure 11: number of nodes Vs dropping ratio

\section{Average Energy Consumption:}

The average energy consumption in every scenario for all node densities in ECDSR is low than the DSR and AODV.

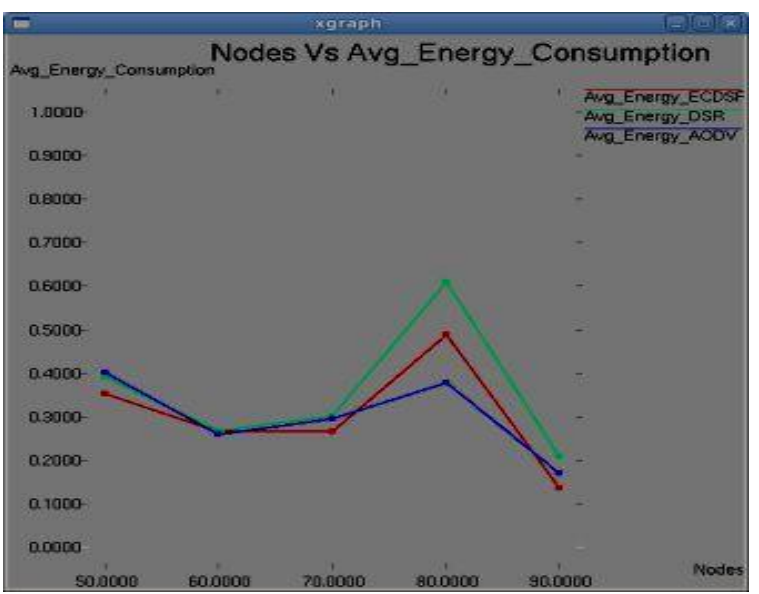

Figure 12: Number of nodes Vs Average energy consumption

\section{Remaining Energy:}

The nodes having lower remaining energy are not participated at the time of communication, so the low energy nodes can be used during crucial condition in future. And the nodes which got select for routing path have higher remaining energy in ECDSR than DSR and AODV.

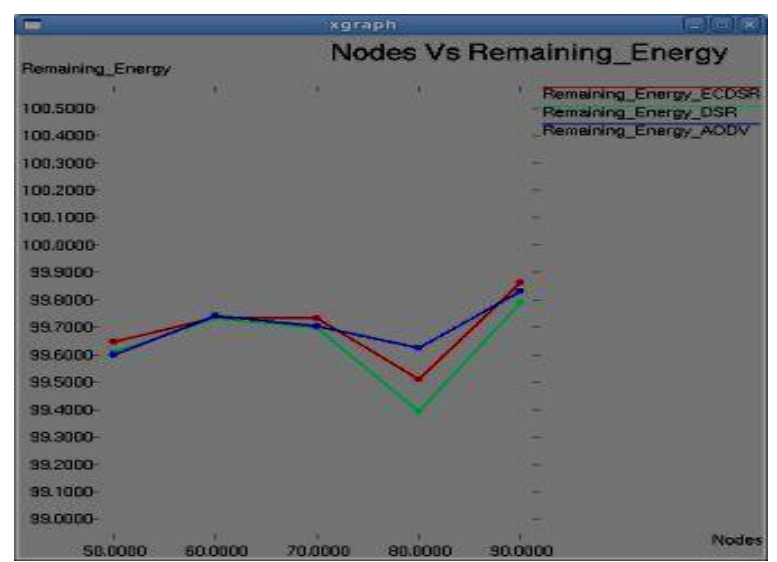

Figure 13: number of nodes Vs Remaining Energy 
Now the following table 2 shows the simulation results for all scenarios with respect to each parameter for ECDSR algorithm.

Table 2: Simulation Results

\begin{tabular}{|c|c|c|c|c|c|}
\hline $\begin{array}{l}\text { Metrics/ } \\
\text { Nodes }\end{array}$ & 50 & 60 & 70 & 80 & 90 \\
\hline $\begin{array}{l}\text { Number } \\
\text { of } \\
\text { packets } \\
\text { send }\end{array}$ & 1801 & 1801 & 1801 & 1801 & 1801 \\
\hline $\begin{array}{l}\text { Number } \\
\text { of } \\
\text { packets } \\
\text { receive }\end{array}$ & 1799 & 1798 & 1757 & 1758 & 1782 \\
\hline $\begin{array}{l}\text { Packet } \\
\text { delivery } \\
\text { Ratio }\end{array}$ & 99.889 & 99.8334 & 97.5569 & 97.6124 & 98.945 \\
\hline $\begin{array}{l}\text { Control } \\
\text { Overhead }\end{array}$ & 124 & 186 & 759 & 2074 & 215 \\
\hline $\begin{array}{l}\text { Normaliz } \\
\text { ed } \\
\text { routing } \\
\text { overhead }\end{array}$ & 0.068927 & 0.103448 & 0.431986 & 1.17975 & 0.120651 \\
\hline Delay & 0.949037 & 0.06119 & 0.102208 & 0.291058 & 0.039031 \\
\hline $\begin{array}{l}\text { Throughp } \\
\text { ut }\end{array}$ & 159912 & 159825 & 156193 & 156352 & 158402 \\
\hline Jitter & 0.100001 & 0.100048 & 0.101252 & 0.102397 & 0.101006 \\
\hline $\begin{array}{l}\text { Number } \\
\text { of } \\
\text { packets } \\
\text { dropped }\end{array}$ & 2 & 3 & 44 & 43 & 10 \\
\hline $\begin{array}{l}\text { Dropping } \\
\text { Ratio }\end{array}$ & 0.111049 & 0.166574 & 2.44309 & 2 & 1.05497 \\
\hline $\begin{array}{l}\text { Total } \\
\text { energy } \\
\text { consum } \\
\text { ption }\end{array}$ & 10.5167 & 9.27396 & 18.378 & 34.1456 & 9.45401 \\
\hline $\begin{array}{l}\text { Average } \\
\text { energy } \\
\text { consum } \\
\text { ption }\end{array}$ & 0.214626 & 0.157186 & 0.266348 & 0.432223 & 0.106225 \\
\hline $\begin{array}{l}\text { Overall } \\
\text { Residual } \\
\text { energy }\end{array}$ & 4889.48 & 5890.73 & 6881.62 & 7865.85 & 8890.54 \\
\hline $\begin{array}{l}\text { Average } \\
\text { residual } \\
\text { energy }\end{array}$ & 99.7874 & 99.8428 & 99.7336 & 99.5678 & 99.8938 \\
\hline
\end{tabular}

\section{CONCLUSION}

The energy efficient routing an important issue in MANET. It is the need of hour for the effective communication in MANET. The various characteristics and issues in MANET are discussed in the paper. In our experiment the mobility of nodes is considered as well as low residual energy which is the cause of route breakage and packet retransmission is solved. By using alert message a node informs the source node to find a new path before any route breakage occurs and hence the established communication continues without any interruption. Also the low energy node get survive for any crucial work in future. The overuse of control information for communication slightly degrades the energy of nodes which is negligible so it is ignored as difference is quite low. Also from simulation results we found that the ECDSR outperforms
DSR and AODV in performance analysis. The lifetime of network get increased in the ECDSR than DSR.

\section{ACKNOWLEDGMENTS}

Author thanks to Dr. M. V. Deshpande, Associate Dean, Mukesh Patel School of Technology Management \& Engineering, NMIMS, Shirpur, for his valuable guidance in performing this project work.

\section{REFERENCES}

[1] Tarique, Mohammed, Kemal E. Tepe, and Mohammad Naserian. "Energy saving dynamic source routing for ad hoc wireless networks." In Modeling and Optimization in Mobile, Ad Hoc, and Wireless Networks, 2005.

[2] Tanque, Mohammed, and Rumana Islam. "Minimum Energy Dynamic Source Routing Protocol for Mobile Ad Hoc Networks." (2007).

[3] De Rango, Floriano, Paola Lonetti, and Salvatore Marano. "MEA-DSR: A Multipath Energy-aware Routing Protocol for Wireless Ad Hoc Networks." In Advances in Ad Hoc Networking, pp. 215-225. Springer US, 2008.

[4] Talooki, V., Hugo Marques, Jonathan Rodriguez, Hugo Água, Nelson Blanco, and Luís Campos. "An energy efficient flat routing protocol for wireless ad hoc networks." In Computer Communications and Networks (ICCCN), 2010 Proceedings of 19th International Conference on, pp. 1-6. IEEE, 2010.

[5] Zhu, Jinhua, and Xin Wang. "Model and protocol for energy-efficient routing over mobile ad hoc networks." Mobile Computing, IEEE Transactions on 10, no. 11 (2011): 1546-1557.

[6] Baisakh, and Nileshkumar R Patel. "Energy Saving and Survival Routing Protocol for Mobile Ad Hoc Networks." International Journal of Computer Applications 48, no. 2 (2012): 13-17.

[7] Patel NileshkumarR., and Shishir Kumar. "Energy Conscious DSR in MANET." In Parallel Distributed and Grid Computing (PDGC), 2012 2nd IEEE International Conference on, pp. 784-789. IEEE, 2012.

[8] Sisodia, Sheetal, and SandeepRaghwanshi. "Performance evaluation of a Table driven and On-Demand Routing protocol in Energy Constraint MANETs." In Computer Communication and Informatics (ICCCI), 2013 International Conference on, pp. 1-8. IEEE, 2013.

[9] Kumari, Anu, Arvind Kumar, and Akhil Sharma. "Survey Paper on Energy Efficient Routing Protocol in MANET." International Journal 3, no. 3 (2013).

[10] Gumaste, S. V., and M. U. Kharat. "Performance Analysis of DSR Protocol", International Journal of Scientific Engineering and Research (IJSER), ISSN (Online): 2347-3878, Volume 1, Issue 3, pp. 62-68, November 2013.

[11] Kumar, Ravi, and Prabhat Singh. "Performance analysis of AODV, TORA, OLSR and DSDV Routing Protocols using NS2 Simulation.".

[12] Network Simulator (NS-2), http://www.isi.edu/nsnam/ns/. 